\title{
42 EFFECT OF MUSIC THERAPY AND MEDITATION ALONG WITH CONVENTIONAL PHYSIOTHERAPY MANAGEMENT IN SUB-ACUTE STROKE PATIENTS
}

Shaji John, ${ }^{1}$ G L Khanna, ${ }^{2}$ Priya Kotwal ${ }^{3}$ 'Faculty and PT Research Association, Department of Rehabilitation Sciences, College of Applied Medical Sciences, King Saud University, Riyadh, Kingdom of Saudi Arabia; ${ }^{2}$ Faculty of Applied Sciences, Manav Rachna International University, Faridabad, Harayana, India; ${ }^{3}$ Manav Rachna International University, Faridabad, Harayana, India

\subsection{6/bjsm.2010.078725.42}

Stroke is the third leading cause of death and the most common cause of disability in adults. The conventional approaches used for rehabilitation are Bobath, Brunstrom, Rood, etc and the physiotherapy measures that are often used include positioning, mat exercises, weight bearing, ice and pressure tapping. Music has been shown to enhance well-being, reduce pain, distract patients from unpleasant thoughts and symptoms and affect patients physiologically and psychologically. Meditation is a process of bringing about a state of self-awareness and inner calm and cause relaxation. These activities can improve self-esteem, happiness and day-to-day functioning in patients.

Study design Pre- and postcontrol group design.

Sample 60 subjects ( 38 males, 22 females) aged $50-70$ years were allotted to three groups randomly.

Method The outcome measures included Hamilton Rating Scale for Depression, Berg Balance Scale, Barthel ADL Index and Fatigue Severity Scale. The three groups underwent training for a period of 6 weeks. In addition to conventional management, group A listened to film and classical songs and group B underwent a meditation protocol. Group C served as the control.

Results Disability, balance, fatigue and depression improved in group A by $12(p<0.05), 7.4(p<0.05), 6.4(p<0.05)$ and 5.6 $(p<0.01)$, respectively and in group $B$, improved by $6(p<0.05)$, $2.2(\mathrm{p}<0.05), 3.2(\mathrm{p}<0.05)$ and $5.2(\mathrm{p}<0.01)$, respectively.

Conclusion Music therapy and meditation are more beneficial than conventional physiotherapy management alone and music therapy is found to be the better among them. 